It follows that

$$
\begin{aligned}
\left|\frac{r_{n}}{r_{n}}\right| & <\frac{2 n^{2}+n}{\left(3 n^{2}-1\right)(n+1)^{2}} \\
r_{n}^{\prime} & <\frac{2 n+1}{\left(3 n^{2}-1\right)(n+1)^{2}}
\end{aligned}
$$

( $r_{n}{ }^{\prime}$ and $r_{n}$ are both positive). It follows therefore that the convergence of the method is fairly rapid. If $n=1$ the remainder ratio is $3 / 8$, whereas if $n=10$ it is .006 .

$$
\begin{aligned}
s_{10} & =1.47113 \\
s_{11} & =1.48014 \\
s_{10} & =s_{11}+10\left(s_{11}-s_{10}\right) \\
& =1.5702
\end{aligned}
$$

It will be observed that, because $s_{11}-s_{10}$ is multiplied by 10 , a decimal place of accuracy is lost. Also the figures given for $s_{10}$ and $s_{11}$ may be in error by .5 units of the last place, and so $s_{10}{ }^{\prime}=1.5702 \pm .0001$, it being possible for $s_{11}-s_{10}$ to have an error 1 in the last place. Also $r_{10}{ }^{\prime}<21 / 299 \times 121=.0006$ and is positive. Thus $1.5702-.0001<\pi / 2<1.5702+.0001+.0006$ or $1.5701<\pi / 2$ $<1.5709$, comparing well with the true value 1.5708 . The agreement is remarkably good for the comparative roughness of the approximation.

The first draft of this paper was written while the author was on the staff of the Royal Military College of Science, Shrivenham, and was communicated by kind permission of the Commandant.

University College of North Wales

LL. G. Chambers

Bangor, Wales

1. Sir Harold Jeffreys \& Bertha Swirles Jeffreys, Methods of Mathematical Physics, Cambridge Univ. Press, New York, 1956, p. 265.

\title{
Factors of Fermat Numbers
}

The writer has prepared a multiple precision routine for the SWAC which tests numbers of the form $N=k \cdot 2^{n}+1$ for primeness. If $N$ is prime, it is then tested to find whether it divides any Fermat number $F_{m}=2^{2^{m}}+1$. The running time for either test is about a minute and a half for $n$ near 500 , and about seven minutes for $n$ near 1000 . There is also a preliminary sieve routine which examines $N$ for small factors. If there is no small factor, the smallest positive number $a$ for which $(a / N)=-1$ is found. The congruence $a^{(N-1) / 2} \equiv-1(\bmod N)$ is then a necessary and sufficient condition for primeness, at least if $k<2^{n}[1]$.

During the period September-November 1956, the cases $k=3,5,7$ were run for $n<1024$, and the odd values of $k$ from 9 to 57 were: run for $n<512$. Some isolated larger values of $k$ have also been used. The cases for $k$ up to 17 and the other results listed below have been checked by a second run. The work is con- 
tinuing. The operation of the SWAC has been handled, for the most part, by John L. Selfridge.

Fourteen new factors of Fermat numbers have been found, namely

$$
\begin{array}{rrr}
21 \cdot 2^{41}+1 \mid F_{39}, & 29 \cdot 2^{57}+1 \mid F_{55}, & 9 \cdot 2^{67}+1 \mid F_{63}, \\
7 \cdot 2^{120}+1 \mid F_{117}, & 5 \cdot 2^{127}+1 \mid F_{125}, & 17 \cdot 2^{147}+1 \mid F_{144}, \\
1575 \cdot 2^{157}+1 \mid F_{150}, & 3 \cdot 2^{209}+1 \mid F_{207}, & 15 \cdot 2^{229}+1 \mid F_{226}, \\
29 \cdot 2^{231}+1 \mid F_{228}, & 21 \cdot 2^{276}+1 \mid F_{268,} & 7 \cdot 2^{290}+1 \mid F_{284}, \\
7 \cdot 2^{320}+1 \mid F_{316,} & 27 \cdot 2^{455}+1 \mid F_{452 .} &
\end{array}
$$

Also, the number $N=k \cdot 2^{n}+1$ was found to be prime in just the following cases with $k=3,5,7$ and $20<n<1024$.

$$
\begin{array}{ll}
k=3: & n=30,36,41,66,189,201,209,276,353,408,438,534 \\
k=5: & n=25,39,55,75,85,127 . \\
k=7: & n=26,50,52,92,120,174,180,190,290,320,390,432,616,830 .
\end{array}
$$

After the project is completed, it is planned to publish a list of all the primes found.

University of California

Raphael M. Robinson

Berkeley, Calif.

The work reported was sponsored by the Office of Naval Research.

1. L. E. Dickson, History of the Theory of Numbers, Carnegie Inst. of Washington, v. 1, 1919, p. 92.

\section{REVIEWS AND DESCRIPTIONS OF TABLES AND BOOKS}

1[A].-H. S. UHLER, "Exact values of 996!, and 1000!, with skeleton tables of antecedent constants," Scripta Mathematica, v. 21, 1955, p. 261-268.

This paper gives the values of 996 ! and 1000 ! without the terminal zeros of which there are 246 and 249 respectively. There is also given the exact value of $(996 !+1) / 997$, together with a frequency census of the digits $0-9$ in each of these three large numbers. The last mentioned number is a "Wilson quotient" and the fact that it is an integer constitutes a check on the work. Certain partial products of 25 consecutive integers beyond 750 are also given.

The work was done by desk calculator as far as 750 ! and then finished on the UNIVAC. The reviewer has compared the value of 1000 ! with the result obtained directly on the SWAC by Kenneth Ralston. The agreement was perfect.

University of California

D. H. LEHMER

Berkeley, California

2[A].-S. C. Nicholson \& J. JeEnel, "Some comments on a NORC computation of $\pi, " M T A C$, v. 9, 1955, p. 162-164.

This gives $\pi, 3089 \mathrm{D}$. It also includes frequency counts of the digits in $\pi$.

C. B. T. 